\title{
Early feeding after major non-laparoscopic gynaecological surgery did not increase gastrointestinal symptoms
}

\author{
MacMillan SL, Kammerer-Doak D, Rogers RG, et al. Early feeding and the incidence of gastrointestinal symptoms after \\ major gynecologic surgery. Obstet Gynecol 2000 Oct;96:604-8.
QUESTION: Is an early low residue diet after major non-laparoscopic gynaecological surgery for benign indications associated with increased postoperative ileus or other gastrointestinal symptoms when compared with conventional postoperative dietary management?

\section{Design}

Randomised (unclear allocation concealment), unblinded, controlled trial with follow up at hospital discharge.

\section{Setting}

A university hospital in Albuquerque, New Mexico, USA.

\section{Patients}

150 women who had major abdominal or vaginal nonlaparoscopic gynaecological surgery for benign indications. Exclusion criteria were history of malignancy, inflammatory bowel disease or obstruction, or current or previous surgery involving extensive lysis of bowel adhesions. $93 \%$ of women ( $46 \%$ white, $36 \%$ Hispanic) were included in the final analysis.

\section{Intervention}

67 women received early feeding and were offered a low residue diet within 6 hours of arrival on the ward. 72 women received conventional dietary management of late feeding and received only ice chips in the immediate postoperative period, clear fluids when normal bowel sounds were detected, and a regular diet after passage of flatus or bowel movements.

\section{Main outcome measures}

Main outcome was postoperative ileus (defined as hypoactive bowel sounds, abdominal distension, nonpassage of flatus or bowel movements with or without nausea or vomiting after the first postoperative day). Secondary outcomes included severe or paralytic ileus (symptoms lasting $>24 \mathrm{~h}$ or requiring nasogastric tube insertion), mean duration of intravenous fluid administration, caloric intake on the first postoperative day, mean number of days to passing of flatus and first bowel movement, abdominal symptoms, median pain scores (McGill Pain scale), and pain medication received.

\section{Main results}

Overall incidence of postoperative ileus was $4.4 \%$ and incidence of severe postoperative ileus was $0.7 \%$. The early and late feeding groups did not differ for the incidence of postoperative ileus $(3.0 \%$ v $5.8 \%, \mathrm{p}=0.68)$; severe ileus $(0 \% v 1.4 \%, \mathrm{p}=1.0)$; mean duration of IV fluid administration; caloric intake on the first postoperative day; mean number of days to passing of flatus and first bowel movement; abdominal symptoms of crampy pain, distension, or vomiting; median pain scores on first postoperative day and on day of discharge; and pain medication received. A stratified analysis comparing abdominal and vaginal surgeries found no differences in outcomes. Fewer women who received early feeding complained of nausea $(13 \% v$ $23 \%, \mathrm{p}=0.04)$.

\section{Conclusion}

Early feeding with a low residue diet did not increase gastrointestinal symptoms after major non-laparoscopic gynaecological surgery when compared with conventional later feeding.

\section{COMMENTARY}

It has been shown previously that patients receiving a clear liquid diet the morning after major gynaecological surgery recovered more quickly than those who were fed after the first passage of flatus. ${ }^{1}$ The study by MacMillan $e t$ al adds to our knowledge base by examining this issue in a similar patient population, by feeding even earlier with a more progressive diet.

The authors note that the sample size was calculated using a much higher incidence of postoperative ileus, and thus the study was underpowered to detect differences. The findings must be interpreted carefully; however, the lack of significant differences in other gastrointestinal symptoms such as abdominal distension lends support to the authors' conclusion that the findings are clinically significant in support of early feeding.

Of major clinical importance is the lower incidence of nausea in the early feeding group ( $13 \% v 23 \%$ in the late feeding group). Postoperative nausea and vomiting increase the cost of treatment and may lead to dissatisfaction with the surgical experience, increased anxiety, and anticipatory nausea in the future. ${ }^{2}$ Because of their substantial effects on quality of life, prevention of nausea and vomiting is crucial.

The results are relevant to medical-surgical and women's health nurses, nurse practitioners, and clinical nurse specialists working with surgical patients. The findings increase our understanding of the appropriate dietary management of patients after surgery and continue to challenge the dogma of late feeding. Early feeding with a low residue diet was not associated with an increase in complications and reduced postoperative nausea. As costs of treating nausea are substantial in terms of patient quality of life, drugs, and nursing time, this finding is particularly relevant to nursing practice. Nurses can use this knowledge to advocate for changes in postoperative dietary practices to improve patient care.

\section{Hilaire J Thompson, RN, CRNP, MS Acute Care Nurse Practitioner University of Pennsylvania Philadelphia, Pennsylvania, USA}

1 Cutillo G, Maneschi F, Franchi M, et al. Early feeding compared with nasogastric decompression after major oncologic gynecologic surgery: a randomized study. Obstet Gynecol 1999;93:41-5.

2 Larson P, Halliburton P, DiJulio J. Nausea, vomiting, and retching. In: Carrieri-Kohlman V, Lindsey AM, West CM, editors. Pathophysiological phenomena in nursing: human responses to illness. Second edition. Philadelphia: WB Saunders, 1993:371-94.
Source of funding: NCRR-GCRC.

For correspondence: Dr D Kammerer-Doak, Women's Health Services, Lovelace Healthcare Center (Journal Center) 5150 Journal Center Boulevard NE, 1st Floor, Albuquerque, $N M$ 87109 , USA. Fax +1 5052623198 . 